\title{
Tumoral pericardial involvement in non-Hodgkin Iymphoma-spektrum of CT findings
}

\author{
Anca Filip Flintoaca ${ }^{1}$, Magdalena Ichim¹, Dana Tabac ${ }^{1}$, Ioana Gabriela Lupescu ${ }^{1,2}$
}

'Department of Radiology, Medical Imaging and Interventional Radiology, Fundeni Clinical Institute, Bucharest "'Carol Davila" University of Medicine and Pharmacy, Bucharest, Romania

\section{ABSTRACT}

Lymphomatous involvement of pericardium is relatively commonly seen in patients diagnosed with Hodking and non-Hodgking disease, presenting large mediastinal mass. Pericardial and cardiac involvement may be a late manifestation of lymphoma. Pericardial nodular thickening or effusion, a tumoral mass extending into the superior pulmonary veins are signs of secondary tumoral pericardial involvement. But pericardial effusion is not a specific and reliable sign of tumoral pericardium, because in patient with lymphoma can be seen postradiotherapy or in case of infection. Familiarity with imaging aspects in lymphomatous pericardial involvement is essential for an accurate diagnosis.

Key words: pericardial tumor, non-Hodgkin lymphoma, pericardial effusion, imaging signs

\section{INTRODUCTION}

Pericardial involvement in a non-cardiac tumoral process is more frequent seen than primary pericardial/cardiac tumor (1). Local extension, hematogenous / lymphatic dissemination and a transvenous route are the main pathways for tumoral spread (2). Lymphoma, lung and breast carcinoma are more likely to interest pericardium in a tumoral processes $(2,3)$. Computed tomography is the most current imaging method used to detect mediastinal mass and chest disease in patient with known lymphoma $(4,5)$.

\section{CASE REPORT}

We present a case of a male patient, 36 years old, diagnosed with nonHodgkin lymphoma, complaining of retrosternal pain, aggravated by profound inspire and change of patients position.

Contrast-enhaced CT images show a multilobulated, heterogenous large right anterolateral mediastinal mass (figure 1), plated on the right cardiac border with obliteration of the normal tissue planes between the pericardium and the tumoral mass, with flattening of the lateral border of right atrium (figure $1 \mathrm{c}$ ). A
Corresponding author: loana G Lupescu, MD, PHD Anca Filip-Flintoaca, MD, PhD student Department of Radiology Medical Imaging and Interventional Radiology, Fundeni Clinical Institute Bucharest, 258 Fundeni Street, Romania E-mail: ilupescu @gmail.com fillip.anca@gmail.com 


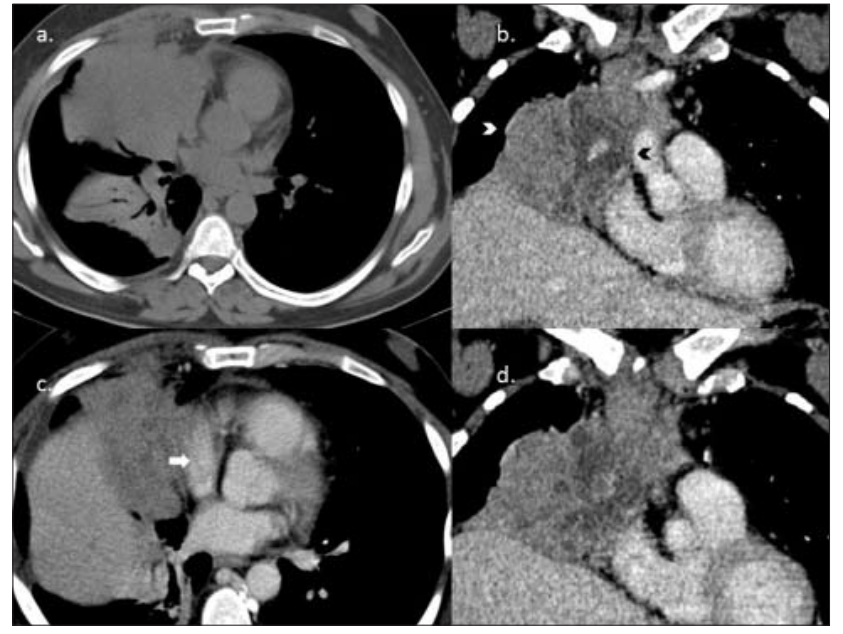

Figure 1 - Large right anterolateral mediastinal mass (arrow heads), plated on right cardiac border, with flattening of right atrium (arrow)

small amount of pericardial effusion (figure $2 a-b$ ) and nodular thickening of right pericardium were noted in the proximity of the tumoral mass (figure $2 c-d$ ). Coronal CT images depicts a large tumoral thrombus (figure $3 \mathrm{~b}$ ) into the superior vena cava (SVC), with almost complete obstruction of SVC (figure $3 a$ ). Also, we noted tumoral encasement and narrowing of right superior pulmonary vein (figure $3 c, d$ ).

Two months postchemoterapy, the tumoral mass

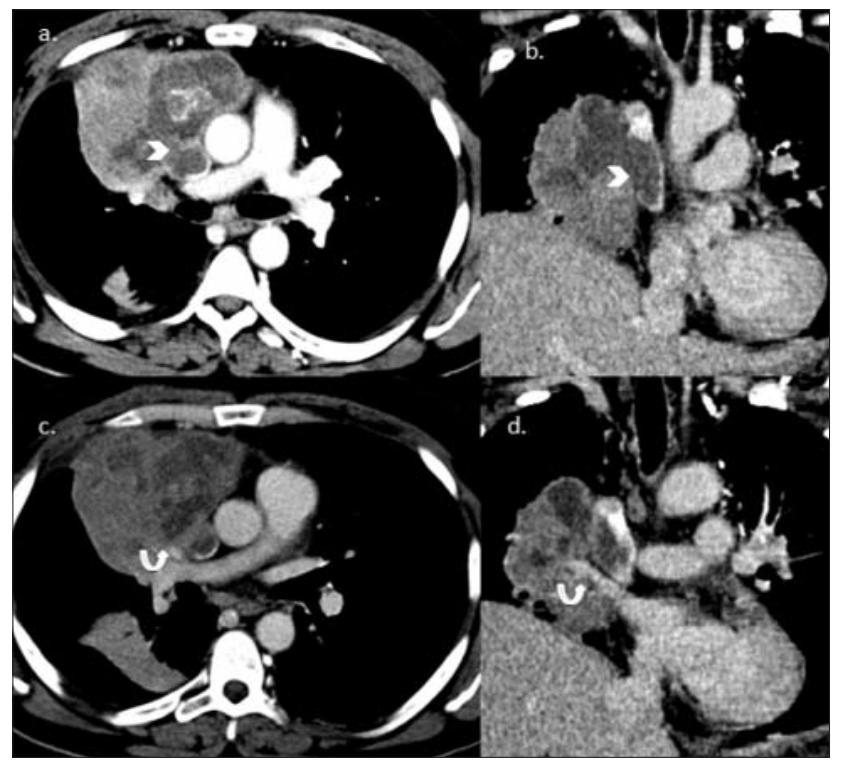

Figure 3 - Direct invasion and intraluminal trombus with narrowing and compression of superior vena cava (a, b-arrow head). Tumoral encasement and narrowing of superior right pulmonary vein (c, d-curved arrow)

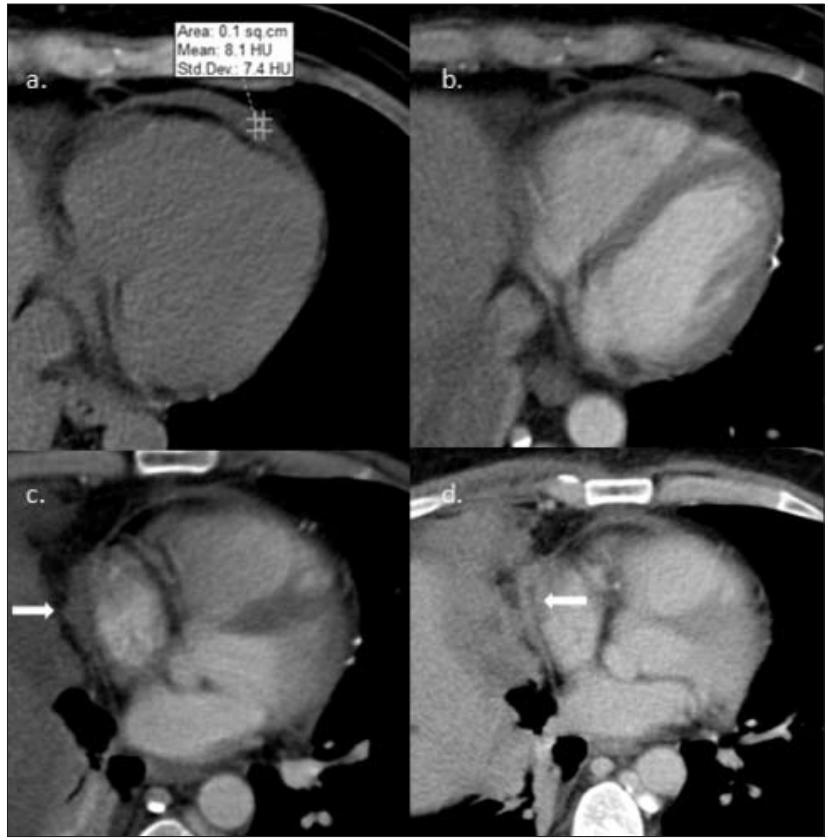

Figure 2 - Small amount of pericardial effusion (a, b) and nodular thickening of pericardial layers (c,d-arrows)

regressed (figure $4 a$ ), with a residual small tumoral thrombus into the distal part of the SVC (figure $4 \mathrm{c}$ ). Also, CT images no longer depict effusion or nodular thickening of pericardium (figure $4 \mathrm{~b}$ ). We noted the development of pulmonary embolism (figure $4 c, d$ ).

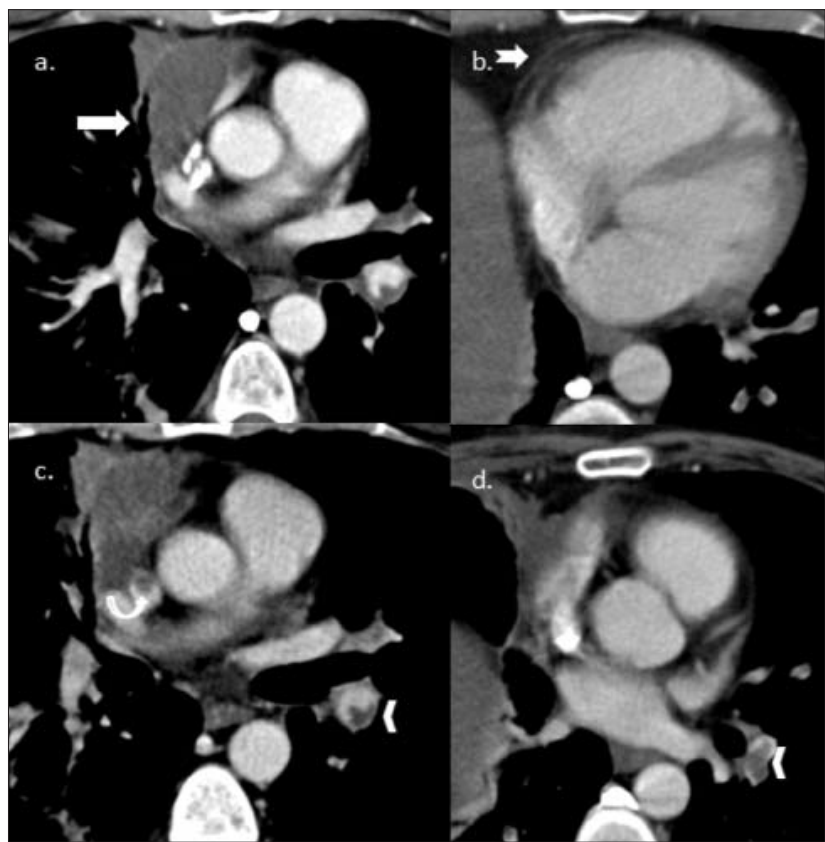

Figure 4 - CT evaluation after chemotherapy. Regression of the tumoral mass (a-arrow) and of the tumoral SVC thrombus (c-curved arrow). Complete regression of pericardial thickening and effusion (b-notched arrow). Emboli involving left inferior pulmonary artery and ramifications 


\section{DISCUSSION}

Secondary tumoral involvement of pericardium is suggested by a pericardial effusion, nodular or diffuse pericardial thickening and a tumoral mass arising or contiguous with the pericardium (6). In a patient diagnosed with lymphoma and pericardial effusion, the differential diagnose includes not only a malignant pericardial disease, but also some other condition (complication of irradiation, opportunistic infection) (7), so is mandatory that the diagnosis to be made by means of pericardiocentesis (2). In our case we showed complete regression of nodular thickening and pericardial effusion postchemotherapy.

Direct extension of the tumoral mass into pericardium is most frequent seen in patients diagnosed with lung carcinoma and lymphoma (2). Also invasion of the superior vena cava and pericardium by a large mediastinal mass is relatively commonly seen (4). In our case we showed a multilobulated mediastinal mass with tumoral thrombus invasion into SVC, with almost complete vein obstruction. Lung carcinoma and lymphoma are the most frequent neoplasms associated with superior vena cava syndrome. Also, we noted flattening of the lateral border of the right atrium. Compression of the cardiac chambers is a finding the may indicate cardiac tamponade. (7). In our case we didn't find any other signs that can suggest cardiac tamponade (enlargement of SVC and IVC, periportal edema etc).

\section{CONCLUSIONS}

Familiarity with the $\mathrm{CT}$ findings in pericardial involvement by lymphoma is essential for an accurate diagnosis and impacts the patient's treatment. Contrastenhanced CT plays a main role in the evaluation of mediastinal mass, tumoral extension, to identify signs of pericardial tumoral involvement or signs of complications.

\section{REFERENCES}

1. Mary L.Grebenc et al. Primary cardiac and pericardial neoplasm: radiologic-pathologic correlation. RadioGraphics 2000; 20:1073-1103.

2. Caroline Chiles et al. Metastatic involvement of the heart and pericardium: CT and MRI imaging. Radiographics (2001) 21:439-49.

3. Kiyoshi Mukai et al. The incidence of secondary tumors of the heart and pericardium: a 10-year study. Jpn.J.Clin.Oncol.18:195-201, 1988

4. Ali Guermazi et al. Extranodal Hodgkin Disease: spectrum of disease. Radiographics 2001, 21:161-179

5. Paolo Toma et al. Multimodality imaging of Hodgkin disease and nonHodgkin lymphomas in children. Radiographics 2007; 27: 1335-1354

6. C.Santiago Restrepo. Imaging findings in cardiac tamponade with emphasis on CT. Radiographics 2007; 27: 1595-1610

7. Venkata S.Katabathina et al. Imaging of oncologic emergencies: what every radiologist should know. Radiographics 2013; 33:1533-1553 